\title{
Electromagnetic Nonlinear Modeling of Active Phased Antenna
}

\author{
G. ZAKKA EL NASHEF, F. TORRES, S. MONS, T. MONÉDIÈRE, T. REVEYRAND, M. THEVENOT, E. \\ NGOYA, R. QUERE \\ OSA/ C2S2 department \\ XLIM CNRS n6172 \\ Limoges, France \\ Email: georges.zakka-el-nashef@xlim.fr
}

\begin{abstract}
This paper examines the distinctive feature of studying the direct interaction between high power amplifiers located at the vicinity of the radiating elements without the use of isolators. In that case, the coupling effect between antennas, which leads to output impedance mismatching and degradation of the radiation pattern, will be described. Alongside, two methods have been developed to address this major drawback with some experimental results to validate the theoretical approach. An active antenna prototype including power amplifiers has been manufactured to validate a first approach of active antenna joint simulation, taking into account all the mentioned effects.
\end{abstract}

Keywords- Active antenna; Electromagnetic interaction; Impedance mismatch; Mutual coupling;

\section{INTRODUCTION}

Active antennas have the distinctive feature of having of integrating, in transmission mode, power amplifiers (PA) located at the vicinity of the radiating elements. Therefore, some major perturbations must be taken into account such as, the mutual coupling related to the antennas array and the mismatching effect related to the PA. However, the integration of active circuitry into antennas gives many advantages in comparison with conventional antennas, especially at microwave and millimeter-wave frequencies. In one hand, the advantages could be reducing the losses and parasitic coupling at higher frequencies, increasing the antenna bandwidth and improving the impedance matching. On the other hand, using active circuitry in large phased arrays can eliminate the need for a complex RF distribution network and a large number of phase shifters as well as for complicated control electronics. Finally, beamforming, nulling patterns, beam steering, directivity enhancement and many more [1], [2] could be achieved by using this circuitry.

Nevertheless, the focus in this paper will be about the TX module, especially the direct interaction between the PA and the antenna. In order to be more explicit about the challenge here, mutual coupling between antennas might lead to an output impedance mismatch and a degradation of the radiation pattern if no isolator is used between them, e.g. a part of the energy radiated by each antenna is captured by the nearby accesses. If isolators do not dissipate this coupled energy, it

This work was realized within the framework of 'Lypsis project', labeled by Elopsys cluster (competitiveness cluster for high technologies in Limousin France - http://www.elopsys.fr), and supported by the French "Direction Générale des Entreprises". could interfere with the PAs, which are connected to the antenna ports. Consequently, it affects the radiation pattern and may damage the efficiency.

In order to model and simulate active arrays in a computationally efficient way, a specific approach should be used. Therefore, a joint simulation tool is developed to take into account, in one hand, the mutual coupling between antennas and, on the other hand, the mismatching effects in PA. Section II is dedicated to the EM macromodel brief description with some experimental validations. Section III is reserved for the behavioral modeling of $\mathrm{PA}$, in order to solve the mismatching problem. A bilateral behavioral model of PA has thus been developed and it will eventually be able to take into account strong load mismatch (VSWR = 4). Some experimental results will be shown as well. Finally, section IV will present the experimental validation of a preliminary approach of active antenna joint simulation tool using an active antenna prototype.

\section{ANTENNA ARRAY MACROMODELING}

\section{A. Brief description}

Antennas are spatially distributed structures and radiation effects are of great importance for them. One cannot ignore the radiation effects during the antenna analysis, because radiation is the primary antenna function. One of the main difficulties in designing and modeling antenna array consists in reaching the highest efficiency for the design and avoiding some phenomena like radiation degradation, especially when couplings are strongly affecting the performances. Furthermore, the mutual coupling affects the antenna access impedance $(\neq 50 \Omega)$ in function of the frequency and the pointing angle, which leads to load variations at the amplifiers outputs. To be more explicit, the mutual coupling has a strong influence on the PA in term of gain (AMAM - magnitude difference between the output and the input) and phase (AMPM - phase difference between the output and the input): the necessary weights applied to each antenna of the array for a given pointing direction will be modified once fed to the PA, which might affect their transfer characteristics and modify the overall performance of the array [3]. Therefore, accurate 
modeling methods are representing a key factor for a successful design.

In this paragraph, we will present a brief description about the development of the EM approach. More details can be found in [3]. To start developing the EM approach, we consider, as an example, a standard linear array of 8 patch antennas working at $8.2 \mathrm{GHz}$ with an inter-element spacing of $0.4 \lambda_{0}$ in order to increase the coupling effect $\left(S_{i j} \approx-13 d B\right)$ and observe the influence on the matching impedances. First of all, a full-wave 3D EM simulation was carried out using CST Microwave Studio (MWS). This EM analysis provides a complete voltage $[U]$ and current [I] matrix and radiated farfield patterns $\left(E_{i}(\theta, \phi)\right)$ of the 3D EM structure. [U] and [I] matrixes respectively represent the simulated driven and parasitic voltages and currents of each antenna in the array. Using these simulated data, the input impedance $\left[Z_{e}\right]$ and the coupling coefficients $\left[\alpha_{i i}\right]$ are computed using the following equations respectively, where $\left[Z_{g}\right]$ is the intrinsic impedance of each feeding port used in CST:

$$
\begin{aligned}
& {\left[Z_{e}\right]=\frac{\left[Z_{g}\right] \times[U]}{\left[Z_{g}\right] \times[I]-[U]}} \\
& {[\alpha]=[I] \times[U]^{-1}}
\end{aligned}
$$

The next step consists in defining an optimal function $\left(\varphi_{\text {optimal }}\right)$ which provides a desired array radiation pattern in terms of gain and side lobe levels (SLL). The earlier computation in CST MWS of the radiation pattern (called "active element pattern") of each antenna within the array, and the use of array synthesis software "SARA" [4], which was developed in our laboratory, enable us to determine this optimal function:

$$
\varphi_{\text {optimal }}=\beta_{1} \varphi_{1}+\beta_{2} \varphi_{2}+\cdots+\beta_{N} \varphi_{N}
$$

The coefficients $\left({ }_{i \ldots N}\right)$ are the elements of the weighting vector. The elements patterns $\left(\phi_{i \ldots N}\right)$ are vectors containing the electromagnetic fields $\left(E_{i}(\theta, \phi)\right)$, and the optimal radiation pattern $\left(\phi_{\text {optimal }}\right)$ can be obtained by combining an array factor with the transfer function of the antennas array. The last step in this EM macromodel is the computation of each antenna access impedance $\left[Z_{\text {access }}\right]$ with their corresponding weight matrix $[a]$ while respecting the matching condition $\left(Z_{g}=\right.$ $\left.Z_{\text {access }}{ }^{*}\right)$. The matching condition enables us to achieve a maximum transfer of energy while ensuring an optimal radiation, which fits the optimal defined function.

$$
\begin{gathered}
{\left[Z_{\text {access }}\right]=[U] \times[([\alpha] \times[U])]^{-1}} \\
{[a]=[U] \times\left(\sqrt{\Re e\left(\left[Z_{\text {access }}\right]\right)} \times\left[Z_{\text {access }}\right]^{-1}\right)}
\end{gathered}
$$

where $\sqrt{\left[Z_{\text {access }}\right]}$ denotes the square root of each matrix element.

Hence, we developed an EM macromodel (implemented in
Matlab), which takes into account the mutual coupling and enables us to obtain the access impedance (Zaccess) presented by each element of the array, and the necessary weights, according to the frequency $\left(f_{0}\right)$ and the pointing angle $\left(\theta_{i}\right)$.

Table I presents a numerical comparison for a $1 \mathrm{X} 8$ array with a pointing angle of $0^{\circ}$ at $8.2 \mathrm{GHz}$, where we compare nominal excitations (magnitude and phase uniform law) to optimum ones (data obtained from the EM macromodel) detailing the most important factors (gain, SLL, total efficiency). The goal of this table is only to show the advantage of using the optimum weights, obtained from the EM macromodel, over the nominal ones and to establish the maximum transfer of energy obtained from the matching condition $\left(Z_{g}=Z_{\text {access }}{ }^{*}\right)$.

TABLE I. COMPARISON BETWEEN THE NOMINAL AND OPTIMUM WEIGHTS FOR $0^{\circ}, \mathrm{f}_{0}=8.2 \mathrm{GHz}$

\begin{tabular}{|c|c|c|c|}
\hline & Gain $(\mathrm{dB})$ & SLL $(\mathrm{dB})$ & $\begin{array}{c}\text { Total } \\
\text { efficiency }(\%)\end{array}$ \\
\hline Nominal & 14.7 & -13.5 & 83.81 \\
\hline Optimum & 12.9 & -40.3 & 93.92 \\
\hline
\end{tabular}

\section{B. Experimental validation}

An experimental evaluation of the performances of the macro-model is made in order to validate the EM approach and to show efficient radiation patterns (high gain with low SLL) in a given direction $(\theta)$.

In order to accomplish that, we have realized a passive prototype composed of a power divider, phase shifters, attenuators to control the weights, i.e. the magnitude and the phase, associated to $1 \mathrm{X} 8$ antennas linear array working at 8.2 GHz (Fig. 1).

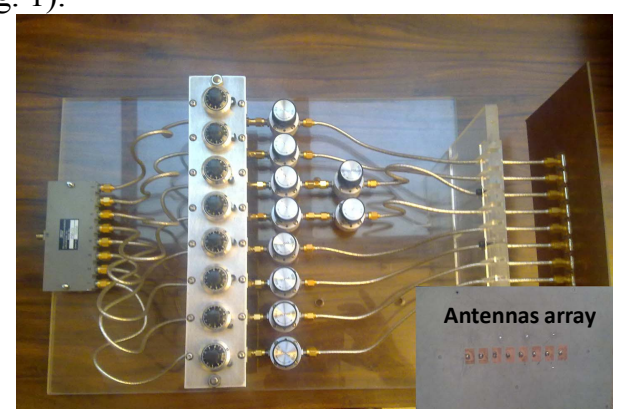

Fig. 1. EM macromodel prototype.

Fig. 2 and Fig. 3 show the comparison between the measurements and the simulations for two different pointing angles $\left(-20^{\circ},+30^{\circ}\right)$. The aforementioned control system, which enables us to control the weights, ensures that the computed weights (magnitudes and phase), obtained from the EM model, are actually set for each port. The working frequency is $8.2 \mathrm{GHz}$. The comparison shows a very good agreement between the simulations and the measurements results. We can conclude that the EM macromodel is efficient and robust. The next paragraph will describe briefly the PA behavioral model with some experimental results. 


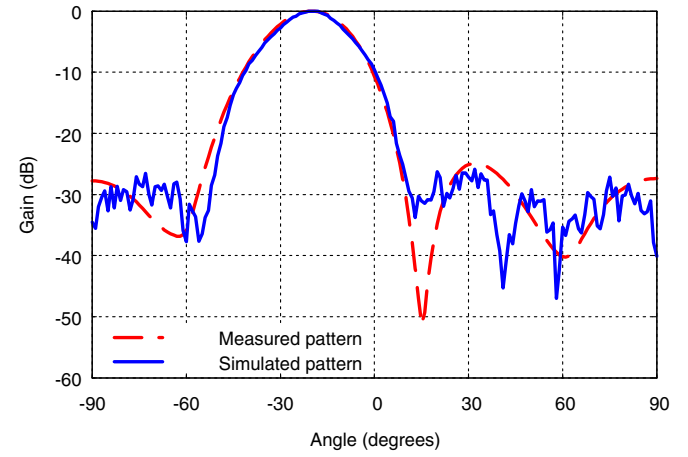

Fig. 2. Radiation patterns comparison for the $1 \times 8$ antennas array, $-20^{\circ}$ pointing angle, $\mathrm{f}_{0}=8.2 \mathrm{GHz}$. Solid line result was obtained by CST computation. Dashed line result was obtained by measurement.

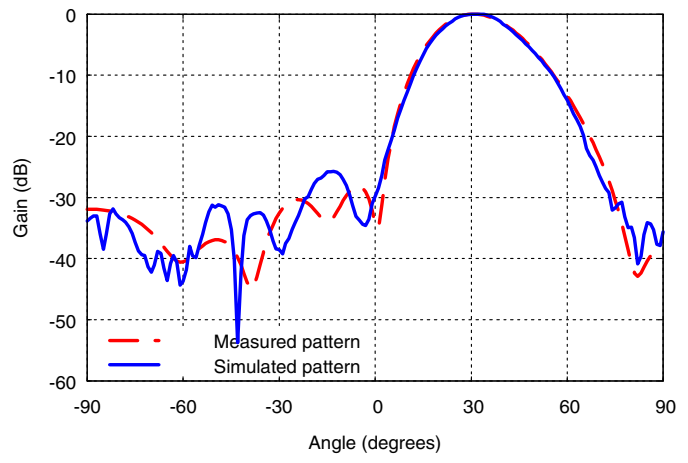

Fig. 3. Radiation patterns comparison for the $1 \mathrm{x} 8$ antennas array, $+30^{\circ}$ pointing angle, $\mathrm{f}_{0}=8.2 \mathrm{GHz}$. Solid line result was obtained by CST computation. Dashed line result was obtained by measurement.

\section{PA NON LINEAR MODELING}

\section{A. Brief description}

PAs are modeled thanks to nonlinear scattering functions [5] that consist in defining a nonlinear relation for the $[S]$ parameters:

$$
\tilde{b}_{i}=\left[S_{i j}\right]_{\text {nonlinear }} \bullet \tilde{a}_{i}
$$

where $\tilde{a}_{i}$ and $\tilde{b}_{i}$ are respectively the incident and reflected power waves at the two ports, and $\left[S_{i j}\right]_{\text {nonlinear }}$ is the nonlinear scattering matrix.

In order to simplify the PA model, we assumed that a PA is considered to work at the fundamental frequency (i.e. $8.2 \mathrm{GHz}$ ) without memory effects. Moreover, since the context of discussion concerns studying the direct interaction between the PA and the antennas $(\neq 50 \Omega)$, e.g. the mismatching effect becomes our major challenge and needs to be taken into account. However, all the models that takes into account the mismatching effect and can be found in the literature are limited to a first order Taylor development, which allows the model to predict the component behavior for small to moderate mismatches (Voltage Standing Wave Ratio $($ VSWR) $=3$ ) at the fundamental frequency. Unfortunately, the reached boundaries concerning the VSWR were not enough, due to the problem of model accuracy working with large VSWR (VSWR=4). Therefore, we expanded the bilateral model to a Taylor development of second order leading to handle high VSWR up to four. All the mathematical formulas and the explanation can be found in [6].

\section{B. Experimental validation}

Extraction and validation of the PA's model have been realized in Agilent ADS in the frequency domain. The model has been implemented thanks to an FDD (Frequency-domain Defined Device) nonlinear block, which allows solving this type of equations in one hand and interpolating extracted large signal S-parameter from measurement files in the other hand. The extraction of the large signal S-parameters has been done using a loadpull bench, allowing us to acquire complete and accurate information of PA electrical behavior. To validate the model and its implementation, we also included a "template" allowing reading measurement data file and plot experimental PA responses for AMAM and AMPM. These responses will be compared to the ones obtained using the black box model (FDD) by realizing HB (Harmonic Balance) simulations.

The fundamental AMAM and AMPM characteristics are compared in Fig. 4 and Fig. 5 respectively for the three extracted impedances corresponding to three different VSWR.

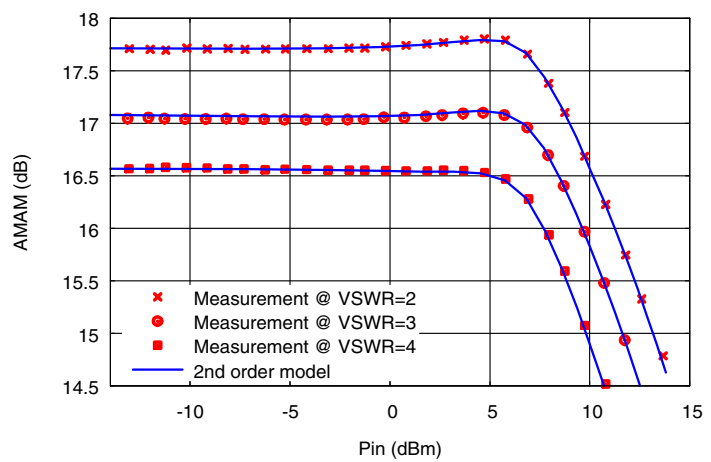

Fig. 4. Fundamental gain compression (AMAM) versus input power for several VSWR level (VSWR $=2 \rightarrow \mathrm{Z} 1=36.8-\mathrm{j} * 27.1$; VSWR $=3 \rightarrow \mathrm{Z} 2=35.2$ $\mathrm{j} * 5.8 ; \mathrm{VSWR}=4 \rightarrow \mathrm{Z3}=14.8+\mathrm{j} * 10.4$ ). Second-order model (lines) compared to measurement (symbols). $\mathrm{f}_{0}=8.2 \mathrm{GHz}$.

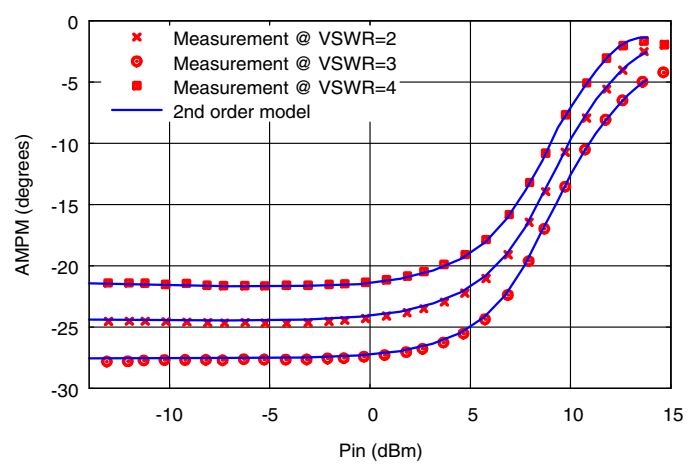

Fig. 5. Fundamental phase variation (AMPM) versus input power for several VSWR level $(\mathrm{VSWR}=2 \rightarrow \mathrm{Z1}=36.8-\mathrm{j} * 27.1 ; \mathrm{VSWR}=3 \rightarrow \mathrm{Z2}=35.2-\mathrm{j} * 5.8$;

$\mathrm{VSWR}=4 \rightarrow \mathrm{Z} 3=14.8+\mathrm{j} * 10.4)$. Second-order model (lines) compared to measurement (symbols). $\mathrm{f}_{0}=8.2 \mathrm{GHz}$.

Fig. 4 and Fig. 5 show an excellent agreement between the PA model and measurements, demonstrating the prediction ability of the PA model to take into account high VSWR up to four. 


\section{JOINT SIMULATION TECHNIQUE}

After the brief description and the experimental validation of both EM macromodel and the PA model, we elaborated a preliminary joint EM/circuit approach that consists of charging the PA by the real impedances, which characterize the antennas, obtained from the EM macromodel. This association between both models will lead to the correction of the modified weights in order to obtain the desired array weighting coefficients. More details can be found in [6]. In this section, we will focus on the experimental validation of the joint simulation approach to demonstrate its' effectiveness. We have realized an active demonstrator based on the one presented in Fig. 1, with the addition of eight PAs directly connected to antennas and without the use of isolators between the antennas and the PAs (Fig. 6). The absence of isolators is related to the PAs, which are able to withstand large VSWR.

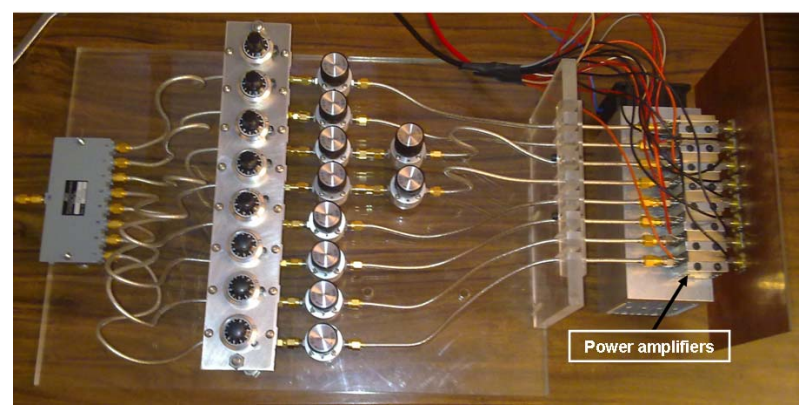

Fig. 6. Active demonstrator

Fig. 7 and Fig. 8 show the comparison between the measured radiation patterns without PA (as in section II), the simulated radiation patterns obtained using CST MWS by a linear combination of the weights calculated by the EM model, and the measured radiation patterns with PA (active demonstrator). A very good agreement between the different radiation patterns configurations can be observed. The main lobes are maintained correctly even with the presence of PA. Some slight differences on the side lobe levels can be noticed, which have been related to some imperfections in the measurement setup.

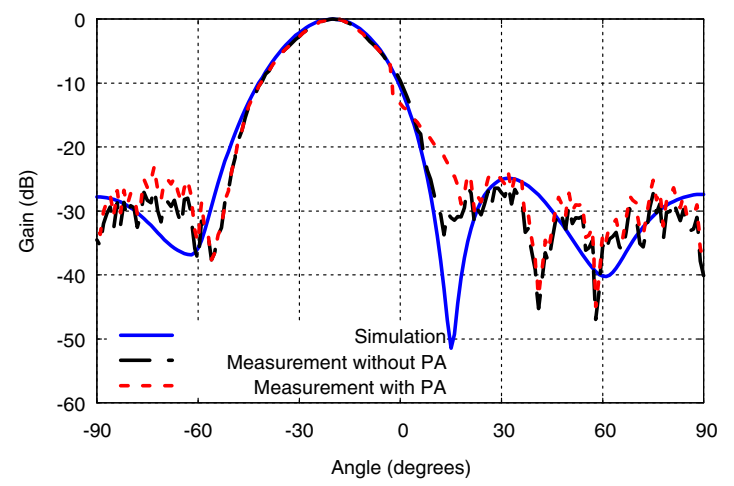

Fig. 7. Radiation patterns comparison $\left(-20^{\circ}\right)$. Active demonstrator measurement (small dashes). Passive demonstrator measurement (no PA) (big dashes). EM macromodel simulation (solid line). $\mathrm{f}_{0}=8.2 \mathrm{GHz}$.

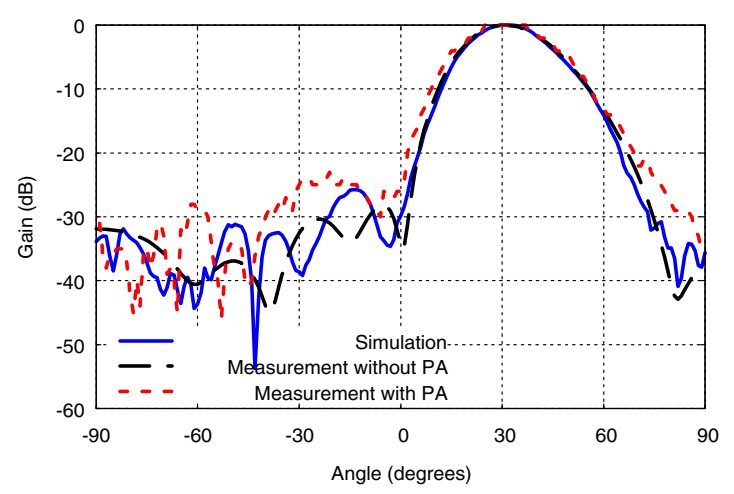

Fig. 8. Radiation patterns comparison $\left(+30^{\circ}\right)$. Active demonstrator measurement (small dashes). Passive demonstrator measurement (no PA) (big dashes). EM macromodel simulation (solid line). $\mathrm{f}_{0}=8.2 \mathrm{GHz}$.

\section{CONCLUSION}

In this paper, we demonstrated the efficiency of an EM macromodel, which considers the mutual coupling and the accuracy of a PA model, which takes into account large VSWR up to four. Both models led to the validation of an active antennas joint simulation approach providing more robustness and accuracy for active antenna design.

\section{References}

[1] H. Hommel, H.P. Feldle, "Current status of airborne active phased array radar system and future trends", 34th European Microwave Conference, Amsterdam 2004

[2] L.C. Godara, "Application of antenna arrays to mobile communications, part I: performance improvement feasibility, and system considerations," Proc. of IEEE, 85, 7, July 1997, pp.1031-1060.

[3] Marc Thevenot, Cyrille Menudier, Ahmad Elsayed Ahmad, Georges Zakka El Nashef, Faycel Fezai, Yasser Abdallah, Eric Arnaud, François Torres and Thierry Monediere, " Synthesis Of Antenna Arrays And Parasitic Antenna Arrays With Mutual Couplings", Internation Journal of Antennas and Propagation, Vol. 2012, Article ID 309728, 22 pages, 2012.

[4] Mouhamadou M., Vaudon P., Rammal M., "Smart antenna array patterns synthesis: null steering and multi-user beamforming by phase control", Progress In Electromagnetics Research, nº 60 (2006) pp. 95106.

[5] J. Verspecht, "Scattering functions for nonlinear behavioral modeling in the frequency domain", Fundamentals of Nonlinear Behavioral Modeling, Foundations and Applications Workshop, IEEE MTT-S International Microwave Symposium, June 2003.

[6] G. Zakka El Nashef, F. Torres, S. Mons, T. Reveyrand, E. Ngoya, T. Monedière, M. Thévenot, R. Quéré, "An accurate modeling technique for antennas and nonlinear RF power amplifier mixed simulation", International Journal of Microwave and Wireless Technologies, volume 3, issue 6, December 2011, pp. 647-655. 